\title{
Dimensions of structurally stable and critical strange non-chaotic attractors
}

\author{
L N C Adamson* and A H Osbaldestin ${ }^{\dagger}$ \\ Department of Mathematics \\ University of Portsmouth \\ Portsmouth \\ PO1 3HF, UK
}

\begin{abstract}
We provide evidence that the box-counting dimension of a structurally stable strange non-chaotic attractor (SNA) of pinched skew product type is equal to 2 by showing that it has non-negligible area. The argument presented is made more accurate in the study of a piecewise linear SNA. Furthermore we provide evidence that the fractal dimension of a critical SNA is not equal to 2 , but in fact lies between 1 and 2 . We numerically calculate the box-counting dimension for several critical SNAs, providing further evidence to support this conjecture.
\end{abstract}

\section{Introduction}

Strange non-chaotic attractors (SNAs) have been found to occur in a wide variety of quasi-periodically forced systems. They are somewhat paradoxical in nature as the geometry of the attractor is strange (fractal) but the dynamics on the set is stable, meaning that nearby points do not separate over time. A good indicator that the dynamics are non-chaotic is the presence of a non-positive Lyapunov exponent. Although convincing evidence of stability, we note that due to the so-called Perron effect for time-varying linearizations, this is not a sufficient condition for stability of the attractor [15]. For most systems it is hard to show that the attracting set is strange, but if the Lyapunov exponent is negative then one may use the methods presented in [20] to numerically indicate strangeness.

The first example of an SNA was presented by Grebogi et al. in [8] and is given by (2.1)-(2.2) (which is commonly referred to as the GOPY model after the authors), and it will be used as an example in Section 2 to demonstrate our method for calculating the box-counting dimension.

The models under consideration are "pinched skew-products" (a term used by Glendinning in [6]), and undergo a transition to SNA at a critical parameter value via the non-smooth pitchfork bifurcation which we have previously studied in [1]. The work in [1] was motivated by [14], in which the bifurcation was labelled "the blow-out birth" of an SNA. In both papers a renormalization approach is presented and scaling properties of the attractor both at and near the critical point of transition are determined showing its self-similarity for certain choices of initial phase. In [14] an adaptation of the system (2.1)-(2.2) is studied, whereas in [1] we perform the analysis on a more general system based on that studied by Glendinning in [7].

\footnotetext{
*luke.adamson@port.ac.uk

†andrew.osbaldestin@port.ac.uk
} 
The idea behind the method we will use was first demonstrated in [4] and is described at the beginning of Section 2. Essentially, we can examine how the area of the attractor inside a strip of width $\epsilon$ decays asymptotically upon iteration of the system, and if we can show that the remaining area is not negligible in this limit then by the definition of the box-counting dimension (as given in (2.5)) the dimension of the attractor is 2 . This is achieved by analysing the evolution of so-called "wedges" in this strip which propagate ergodically upon iteration of the map and repeatedly delete area from the strip. We question the assumption made in [4] that the wedges widen upon iteration and approach a limiting slope, as the "pinched" nature of the SNA suggests these slopes should be unbounded asymptotically. We therefore re-examine the original idea, but use a different determination of the slopes, which we also believe is more intuitive as the slope now depends on the bifurcation parameter.

It is conjectured in [4] that the information dimension of SNAs is equal to 1. In [9] this conjecture is proven for SNAs which arise from pinched skew-product systems of the form

$$
\begin{aligned}
& x_{n+1}=\tanh \left(\kappa x_{n}\right) \cos \left(2 \pi \theta_{n}\right) \\
& \theta_{n+1}=\theta_{n}+\omega \quad(\bmod 1),
\end{aligned}
$$

for $\kappa>0$ sufficiently large (which is determined by the diophantine $\omega$ ) of which (2.1)-(2.2) is an example. Furthermore, it is proved in [9] that the Hausdorff and pointwise dimension for this type of system are also equal to 1. However, this paper does not make rigorous the argument in [4] regarding the box-counting dimension being 2 . We will provide compelling evidence that the box-counting dimension is 2 for a class of piecewise linear pinched skew-products. As the name suggests, these systems are composed of functions which are piecewise linear.

In Section 2 we begin by describing the basic properties of the GOPY model and then we use our modified approach to the method presented in [4] to indicate that the box counting dimension is 2, using a combination of analytic and numeric arguments. The method we use requires the assumption that locally each piece of the bounding curves is approximately linear and in Subsection 2.1 we show that the argument can be made nearly exact for piecewise linear SNAs. Section 3 shows how the results from Section 2 can be generalised to a class of pinched skew-product systems.

At a critical point in parameter space we have an SNA which is not persistent under a small perturbation, and such an SNA is called a critical SNA. Note that renormalization group approaches have previously been applied to study dimensions in critical situations occurring in circle maps [12] and in Feigenbaum's attractor [13]. In Section 4 we demonstrate that the box-counting dimension for a critical "pinched" SNA should lie between 1 and 2. We give evidence to suggest that at a critical point the area of the attractor inside an $\epsilon$ strip is negligible, and hence the dimension may not be two. In [14] and [1] it is demonstrated that SNAs occurring at critical points are fractal in nature and so the dimension can be bigger than 1.

\section{Structurally stable strange non-chaotic attractors}

Following [8], we give the definition below of a strange non-chaotic attractor:

Definition (Strange attractor, strange non-chaotic attractor). A strange attractor is an attractor which is not a finite set of points and is not piecewise differentiable. A strange non-chaotic attractor (SNA) is a strange attractor which does not exhibit sensitive dependence on initial conditions. Quantitatively, we take the latter to mean that the largest Lyapunov exponent is non-positive.

We also give a definition of a structurally stable SNA and a critical SNA:

Definition (Structurally stable and critical). We refer to an strange non-chaotic attractor (SNA) as structurally stable if it is persistent under a small perturbation of any of the parameters of the underlying system. A critical SNA is an SNA which is not structurally stable.

In Grebogi et al. [8] the existence of SNAs was established for two different systems, the first of which has become 


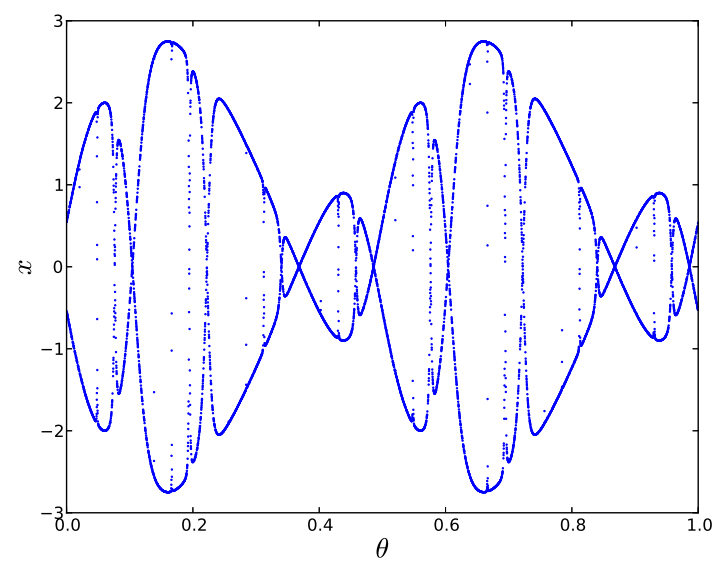

Figure 1: Strange non-chaotic attractor occurring for $\sigma=1.5$ in system (2.1)-(2.2).

known as the GOPY model (after the authors) and is given by

$$
\begin{aligned}
x_{n+1} & =f\left(x_{n}, \theta_{n}\right)=2 \sigma \tanh \left(x_{n}\right) \cos \left(2 \pi \theta_{n}\right), \\
\theta_{n+1} & =\theta_{n}+\omega \quad(\bmod 1) .
\end{aligned}
$$

Here $\omega$ is taken to be irrational and in particular we set $\omega=(\sqrt{5}-1) / 2$, the inverse of the golden mean. The map has two Lyapunov exponents, one of which corresponding to (2.2) is trivially zero, and the other (in the $x$-direction) is given by

$$
\begin{aligned}
\lambda & =\lim _{N \rightarrow \infty}\left(\frac{1}{N} \sum_{i=1}^{N} \ln \left|\frac{\partial f}{\partial x}\right|_{\theta_{i}, x_{i}}\right) \\
& =\int_{0}^{1} \ln \left|\frac{\partial f}{\partial x}\right| \mathrm{d} \theta
\end{aligned}
$$

where the latter equation follows from the ergodicity in $\theta$. A positive Lyapunov exponent is an indication that motion on the resulting attractor (which exists due to the bounded nature of (2.1)) is chaotic, whereas a nonpositive Lyapunov exponent suggests it is stable. In [8] it is analytically proven that $\lambda$ is non-positive for the GOPY model for all choices of $\sigma>0$. Note that a positive (resp. non-positive) exponent is not a sufficient criterion for chaos (resp. stability). See [15] for more details on the so-called Perron effect which can cause sign-inversions of the exponent for time varying linearisations.

In [8] it is shown that for $\sigma<1$ the attractor is simply the circle $x=0$, whereas for $\sigma>1$ we have an SNA. The transition is caused as the circle $\mathcal{L}=\{(\theta, x): x=0\}$ becomes unstable for $\sigma>1$ (verifiable through calculation of its transverse Lyapunov exponent), and thus there must be non-zero $x$. Furthermore, because $\cos (2 \pi \theta)$ is zero for $\theta_{0}=1 / 4$ or $3 / 4, x$ is fixed at zero for all future iterates of these initial points. Due to the ergodicity in $\theta$, the attractor thus contains a dense set of points on $\mathcal{L}$ causing a "pinching" effect, and hence the attractor is nowhere differentiable and thus strange. The critical point $\sigma=1$ will be of interest to us later on. A plot of the SNA for $\sigma=1.5$ is shown in Figure 1.

The SNA is structurally stable (which from now we will refer to as a "stable SNA" for brevity) for $\sigma>1$, and the aim of this paper is to amend and simplify some of the arguments produced in [4] with regard to the box-counting dimension of SNAs. Recall that the box-counting dimension is defined as follows:

Definition (Box-counting dimension). The box counting dimension of an attractor is given by

$$
D_{0}=\lim _{\epsilon \rightarrow 0} \frac{\log N(\epsilon)}{\log 1 / \epsilon}
$$

where $N(\epsilon)$ is the number of boxes of width $\epsilon$ required to cover the attractor. 
In [4] it is conjectured that $D_{0}=2$ for a stable SNA. An argument is presented in support of this result, and involves the following approach. Consider the initial area $\mathcal{A}_{0}$ of the region given by $0 \leq \theta<1,-\infty<x<\infty$. Applying the system (2.1)-(2.2) to it repeatedly produces successive iterates of it which will converge to the attractor. The first four such iterates are shown in Figure 2.
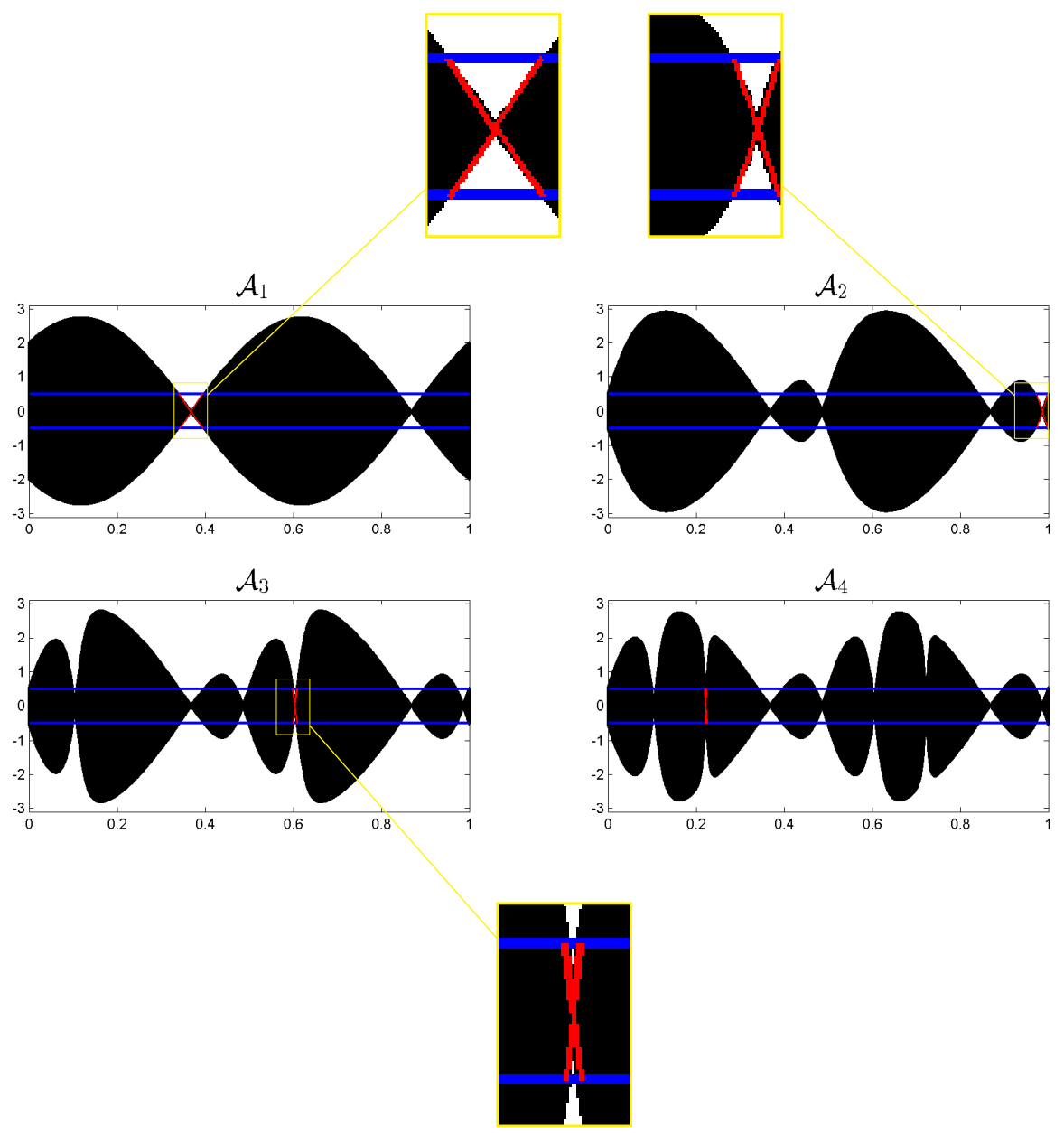

Figure 2: Successive iterates of the initial region $\mathcal{A}_{0}=\{(\theta, x): 0 \leq \theta<1,-\infty<x<\infty\}$ with $\sigma=1.2$. The red lines represent the approximate iterates of the first pair of symmetric wedges whose slopes are given by $(2.7)$.

As these iterations continue, all points will converge towards the attractor, but our interest is in how the remaining area inside an $\epsilon$ strip about the $\theta$ axes decreases as $n \rightarrow \infty$. The initial area of the strip is $2 \epsilon$ and as in [4] our aim is to show that the area deleted from the strip is bounded above by some multiple of $\epsilon^{2}$, in which case the area remaining is non-negligible, which is sufficient for the box-counting dimension to be 2 .

Notice that in the first iterate there are four wedges within the $\epsilon$ strip (shown in blue) and that if $\epsilon$ is sufficiently small the slopes of the edges of these wedges are approximately linear. Each of these wedges stem from the fact that $\cos (2 \pi \theta)=0$ when $\theta=1 / 4$ or $3 / 4$. Indeed, the four wedges appear at the two values of $\theta$ which are the images of $1 / 4$ and $3 / 4$ under $(2.2)$ i.e. $3 / 4+\omega(\bmod 1)=0.368$ and $1 / 4+\omega(\bmod 1)=0.868$ to 3 d.p.

The map is iterated forward and the tips of the wedges are mapped ergodically along the $\theta$ axis, and four new wedges are spawned upon each iteration (corresponding to values of $\theta_{0}$ such that $\left.\theta_{n}= \pm 1 / 4(\bmod 1)\right)$. In [4] it is stated that the number of wedges doubles at each step, which is clearly not the case as can be seen from Figure 2. Furthermore, as $\cos (2 \pi \theta)$ is symmetric about $\theta=1 / 2$, we see that the attractor repeats itself with period $1 / 2$. Letting the (positive) slope of the four initial wedges (which we are assuming is linear for $\epsilon$ sufficiently small) be $\lambda_{0}$ it is trivial to show that the area of a wedge is given by $\epsilon^{2} / \lambda_{0}$. Note that the set of points which map to the tips of 
the wedges is $\mathcal{S}=\{(1 / 4, x)$ or $(3 / 4, x) \mid x \in \mathbb{R}\}$ and so we can see that the maximum possible derivative of equation (2.1) with respect to $\theta$ has magnitude $4 \pi \sigma$ and so $\lambda_{0}=4 \pi \sigma$. We now use elementary straight line geometry to gain a knowledge of how the slopes of the wedges evolve.

Assume the point of the wedge is located at the point $(\alpha, 0)$ (here we assume without loss of generality that the wedge is positive in $x$ ), then taking $\epsilon$ sufficiently small we can see that locally the right hand edge of the wedge is given by the line $x=\lambda_{0}(\theta-\alpha)$. The height of the wedge is $\epsilon$ and so using elementary line geometry we can calculate the two other points on the wedge (or triangle) as shown in Figure 3. To see how the slope evolves we

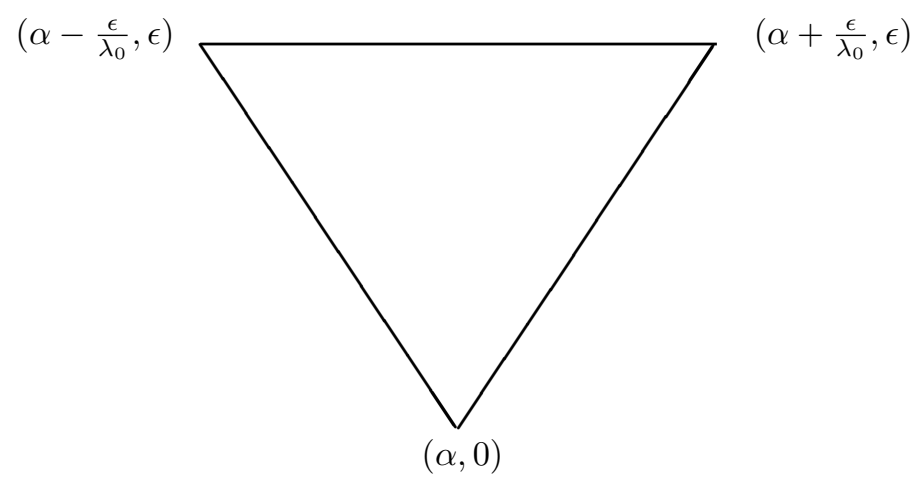

Figure 3: A schematic diagram of a wedge for $\epsilon$ sufficiently small.

simply apply the equations (2.1)-(2.2) to these points to give three new points, which will be the next iterate of the wedge. We then calculate the gradient $\lambda_{1}$ of the new edges in the usual manner giving

$$
\lambda_{1}=\frac{2 \sigma \tanh (\epsilon)\left|\cos \left(2 \pi\left(\alpha+\frac{\epsilon}{\lambda_{0}}\right)\right)\right| \lambda_{0}}{\epsilon} .
$$

We have two equivalent choices for $\alpha$, those being $1 / 4+\omega$ or $3 / 4+\omega$ (which due to the symmetry about $\theta=1 / 2$ of $\cos (2 \pi \theta)$ give rise to the same slope) and so we pick $\alpha=1 / 4+\omega$. As $\epsilon \rightarrow 0$ we have $\tanh (\epsilon) / \epsilon \rightarrow 1$ and iterating forward we thus find the equation of the $n^{t h}$ such iterate of the slope to be

$$
\lambda_{n}=\lambda_{0} \prod_{i=1}^{n} 2 \sigma|\cos (2 \pi(a+i \omega))|,
$$

where $a=1 / 4$. Note that this formula is different to the one produced in [4]. A concern is the balance between the behaviour of how $\epsilon$ must behave as it tends to zero in relation to $n$, and we explore this numerically later on. In Figure 2 the red lines present the linear approximations to successive iterates of the first pair of symmetric wedges obtained using this construction, and shows good agreement. In fact we observe that the linear approximation to the wedges gets closer to the actual wedges as we iterate further, which is indicative of the fact that the wedges asymptote to vertical lines. We would like an understanding of the long term behaviour of $\lambda_{n}$ in order to understand how the area inside the strip evolves, and the following Lemma provides us with this information.

Lemma 1. $S_{n}=\left(\prod_{i=1}^{n}|\cos (2 \pi(a+i \omega))|\right)^{1 / n} \rightarrow 1 / 2$ as $n \rightarrow \infty$.

Proof. Taking the logarithm of the expression converts it from a geometric mean into the arithmetic mean

$$
\mu_{n}=\frac{1}{n} \sum_{i=1}^{n} \ln (|\cos (2 \pi(a+i \omega))|) .
$$

As $n \rightarrow \infty, \mu_{n} \rightarrow \mu$ and, using the ergodicity in $\theta$, we can write

$$
\mu=\int_{0}^{1} \ln |\cos (2 \pi \theta)| \mathrm{d} \theta=-\ln (2) .
$$

Taking the exponential gives $e^{\mu}=1 / 2$ and hence the result follows. 
In Figure 4 we give a plot of $S_{n}$ against $n$ showing the oscillatory nature of the convergence to 0.5 . Numerically we see that $S_{n}>0.5$, which for other values of $a$ is not necessarily the case.

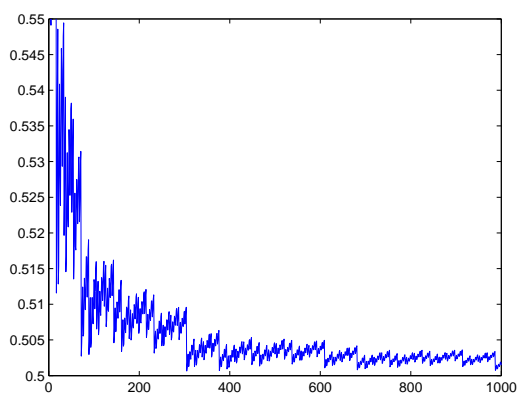

Figure 4: Plot of $n$ vs. $S_{n}$.

A further understanding of the behaviour can be derived from a renormalization analysis. We consider $S_{n}(a)$ at the characteristic times $F_{n}$, where $F_{n}$ denotes the Fibonacci sequence $F_{n+2}=F_{n+1}+F_{n}$ with $F_{0}=0, \quad F_{1}=1$. We have

$$
\begin{aligned}
S_{F_{n+2}}(a) & =\left(\prod_{i=1}^{F_{n+2}}|\cos (2 \pi(a+i \omega))|\right)^{1 / F_{n+2}} \\
& =\left(\prod_{i=1}^{F_{n+1}}|\cos (2 \pi(a+i \omega))| \prod_{i=F_{n+1}+1}^{F_{n+2}}|\cos (2 \pi(a+i \omega))|\right)^{1 / F_{n+2}} \\
& =S_{F_{n+1}}(a)^{F_{n+1} / F_{n+2}} S_{F_{n}}\left(a-(-\omega)^{n+1}\right)^{F_{n} / F_{n+2}}
\end{aligned}
$$

using the fact that $F_{n+1} \omega=-(-\omega)^{n+1}(\bmod 1)$. We now define the rescaled functions

$$
Q_{n}(x)=S_{F_{n}}\left((-\omega)^{n} x+a_{0}\right),
$$

and from (2.12) we see that

$$
Q_{n+2}(x)=Q_{n+1}(-\omega x)^{F_{n+1} / F_{n+2}} Q_{n}\left(\omega^{2} x+\omega\right)^{F_{n} / F_{n+2}},
$$

where $Q_{0}(x)=1, Q_{1}(x)=\left|\cos \left(2 \pi\left(-\omega x+a_{0}+\omega\right)\right)\right|$. Note that we can remove the powers in (2.14) by defining $\hat{Q}_{n}=Q_{n}^{F_{n}}$ which gives the same recurrence for $\hat{Q}_{n}$ obtained in studies of symmetric barrier billiards ([3],[19],[2]), two-level quantum systems ([5],[16]) and the Harper equation ([11], [17]). However, numerically it is better to use the recurrence (2.14) because as discussed above $\hat{Q}_{n}$ will decay like $(0.5)^{F_{n}}$ which means that upon iteration we will be dealing with negligible numbers which will reduce the accuracy (and will fail at the point when the computer identifies them as zero). What we are effectively doing here is factoring out this decay.

Setting $a_{0}=0.25$ as the origin we iterate (2.14) and discover that $Q_{n}$ converges to a scaled period three orbit which is shown in Figure 5.

We see that the value of these functions at the origin (corresponding to $a_{0}=0.25$ ) is always $>0.5$, whereas a small perturbation (due to the fact that we are analysing the function in intervals of decreasing length about $a_{0}$ ) causes the value of the function to drop below 0.5 , and so $a_{0}=0.25$ seems to be a local maximum of $S_{n}(a)$. The value of $Q_{32}(0)$ is 0.500000120963 , which of course corresponds to the value of $S_{n}$ (with $a=0.25$ ) at $n=F_{32}=2,178,309$. Without the renormalization analysis the numerics break down at around $n=1200$ as $S_{n}^{n}$ becomes so small it is identified as zero! Note also that $S_{F_{n}}$ are likely to be the smallest values of this sequence because $1 / 4+F_{n} \omega=1 / 4-(-\omega)^{n} \rightarrow 1 / 4$ as $n \rightarrow \infty$, and so $\cos \left(2 \pi\left(1 / 4+F_{n} \omega\right)\right) \rightarrow 0$.

We can also approximate the scaling factor for the convergence using this approach. We do this by defining $\tilde{Q}_{n}=Q_{n}-0.5$ which represents the deviation of $Q_{n}$ from 0.5 at time $F_{n}$. Examining the sequence $\tilde{Q}_{n}(0) / \tilde{Q}_{n+3}(0)$ 

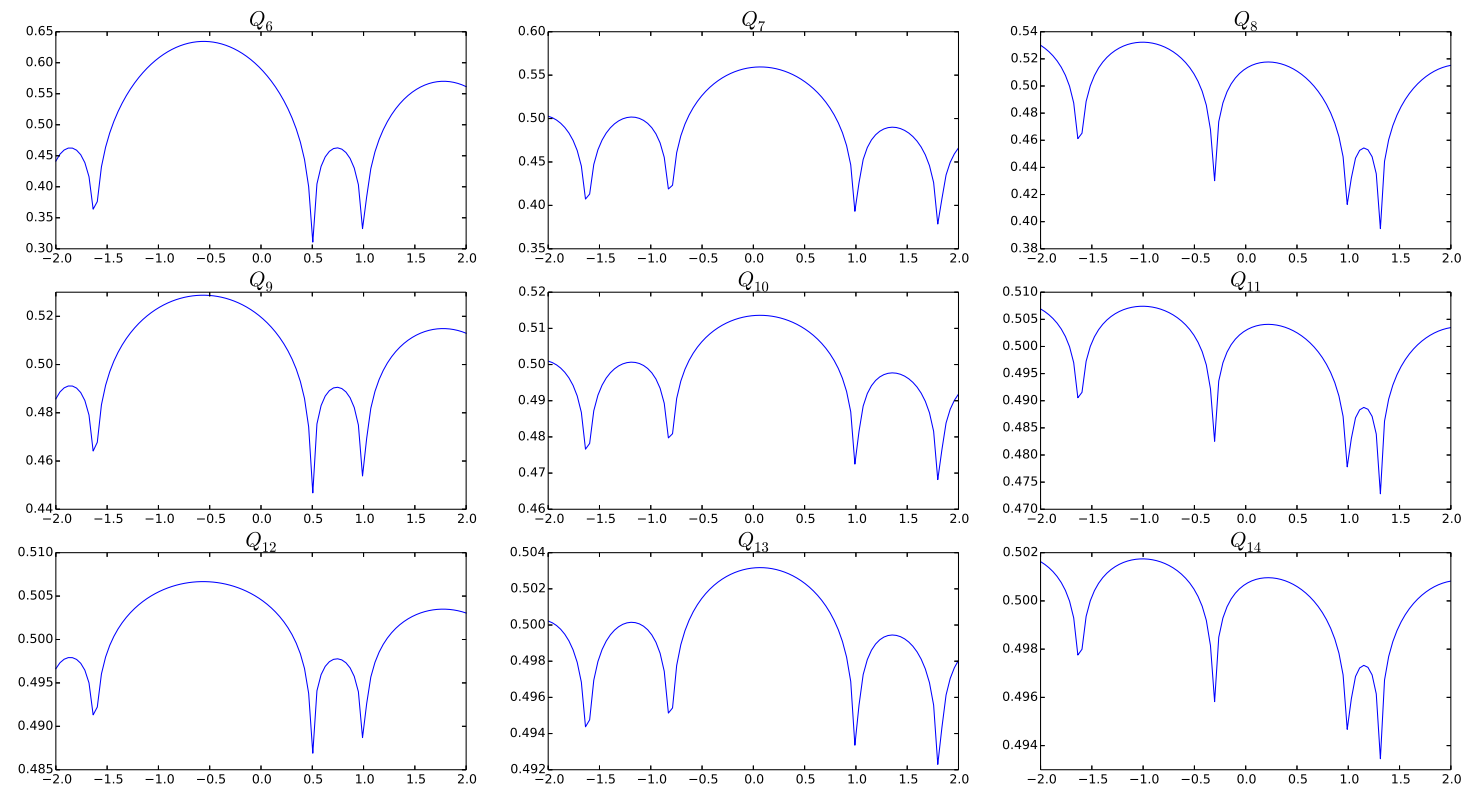

Figure 5: Successive iterates starting with $Q_{6}$ showing clear repetition of the decaying oscillations about 0.5.

we see convergence to a constant value of $\nu=\omega^{-3}$. Hence the deviation from 0.5 decays with exponent $\gamma=$ $\ln \left(\omega^{-3}\right) / 3 \ln (\omega)=-1$. Thus the deviation decays proportional to $n^{-1}$ as shown on the logarithmic scale in Figure 6 .

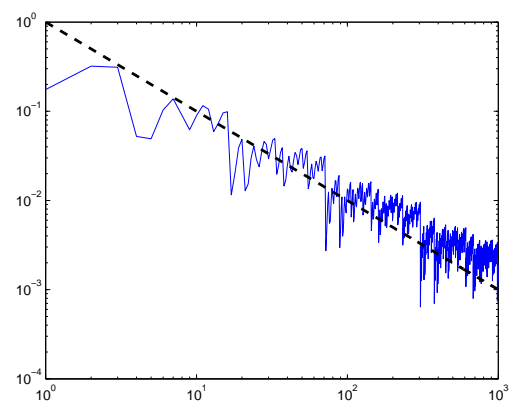

Figure 6: Plot of the deviation of the sequence $S_{n}$ from its limiting value of 0.5 , and the theoretical decay rate $n^{-1}$ (dashed line).

From Lemma 1 we can write

$$
\begin{aligned}
\lambda_{n} & =\lambda_{0} 2^{n} \sigma^{n}(0.5+\tilde{\epsilon}(n))^{n} \\
& =\lambda_{0} \sigma^{n}(1+\underbrace{2^{n}\left(\sum_{k=1}^{n}\left(\begin{array}{l}
n \\
k
\end{array}\right) 0.5^{n-k} \tilde{\epsilon}(n)^{k}\right)}),
\end{aligned}
$$

and for large $n$ this causes substantial growth of the "error" term as $\tilde{\epsilon}(n) \sim n^{-1}$. In particular we can write the error term given in the under-brace of (2.16) as being proportional to the function

$$
\xi(n)=2^{n}\left(\left(0.5+\beta n^{-1}\right)^{n}-0.5^{n}\right),
$$


where $\beta$ is a constant. Logarithmic differentiation gives us

$$
\xi^{\prime}(n)=\frac{\left((n+2 \beta) \ln \left(\frac{n+2 \beta}{n}\right)-2 \beta\right)\left(\frac{n+2 \beta}{n}\right)^{n}}{n+2 \beta} .
$$

Our aim is to prove this is positive for all $n \geq 0$. Note that $t(n)=(n+2 \beta) \ln \left(\frac{n+2 \beta}{n}\right)$ is a monotonic decreasing sequence as its derivative $t^{\prime}(n)=\ln \left(1+\frac{2 \beta}{n}\right)-\frac{2 \beta}{n}<0$. Hence if $(n+2 \beta) \ln \left(\frac{n+2 \beta}{n}\right)$ has a limit it will tend to it from above. Taking the exponential of this expression we can calculate instead the limit

$$
\lim _{n \rightarrow \infty}\left(1+\frac{2 \beta}{n}\right)^{n+2 \beta}=e^{2 \beta}
$$

through the definition of the exponential function. Thus $(n+2 \beta) \ln \left(\frac{n+2 \beta}{n}\right) \rightarrow 2 \beta$ from above and so $\xi^{\prime}(n)$ is positive for all $n \geq 0$. Hence $\xi(n)$ is an increasing function of $n$ for $n \geq 0$. In fact $\xi(n)$ tends to a constant which we can calculate as

$$
\begin{aligned}
\lim _{n \rightarrow \infty} 2^{n}\left(\left(0.5+\beta n^{-1}\right)^{n}-0.5^{n}\right) & =\lim _{n \rightarrow \infty}\left(1+\frac{2 \beta}{n}\right)^{n}-1 \\
& =e^{2 \beta}-1 .
\end{aligned}
$$

The consequence of all these results is clear: as $n \rightarrow \infty$ we see that

$$
\lambda_{n} \sim \sigma^{n} .
$$

This suggests a different asymptotic behaviour than that described in [4], as for $\sigma>1$ there is no limiting slope as the slopes become unbounded. To demonstrate this result, we provide in Figure 7 plots of the partial products $p_{n}=\prod_{i=1}^{n} 2 \sigma|\cos (a+i \omega)|$ (in blue) against the graph of $\sigma^{n}$ (shown in black) for $\sigma=0.99$ and $\sigma=1.01$. Note that as described numerically above $S_{n}>0.5$ for all $n$, and so $\sigma^{n}$ is a lower bound for $p_{n}$. Indeed we have observed that $S_{n}>0.5$ for $n$ up to 1200 and that $S_{F_{m}}>0.5$ up to $m=32$ using the renormalization approach.

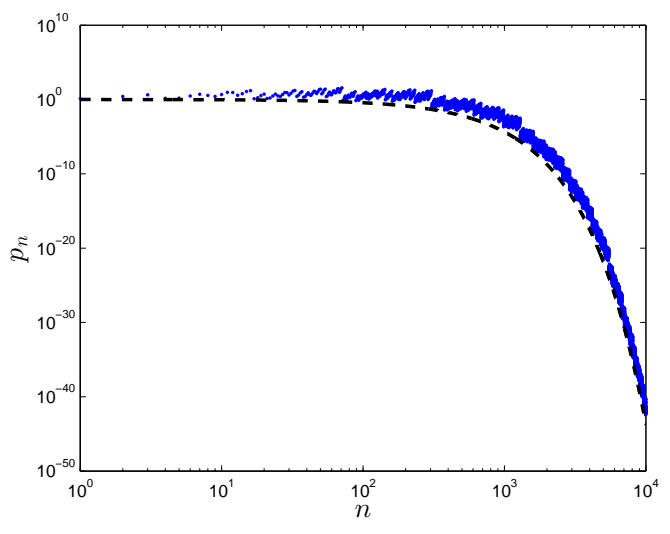

(a) $\sigma=0.99$

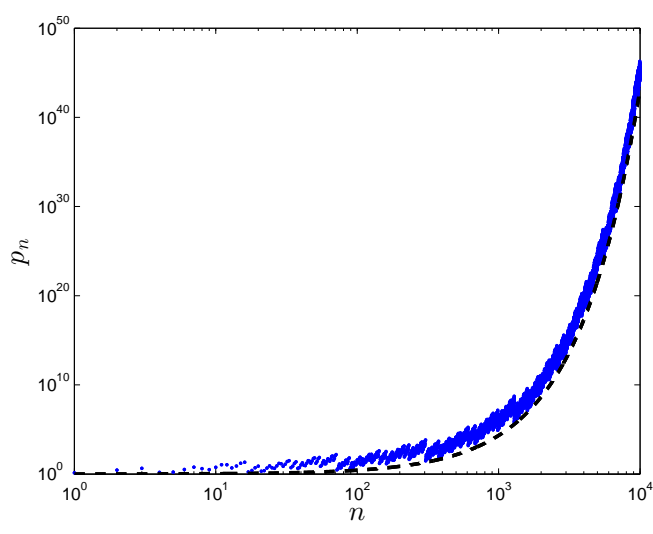

(b) $\sigma=1.01$

Figure 7: Plot of $n$ vs $p_{n}$ and the limiting curve $\sigma^{n}$ (dashed).

The behaviour (2.22) is to be expected because for $\sigma<1$ the attractor is just $x=0$ and so the gradients should be asymptotically zero, whereas for $\sigma>1$ the attracting curve is given by a pair of symmetric non-zero semi-continuous curves [10] and the attractor must be zero on a dense set of points on the $\theta$ axis, and thus we expect the slope at these points to be arbitrarily large and the wedge to turn into a vertical line asymptotically.

Now we examine the effect this has on the area deleted from the $\epsilon$ strip. Firstly, we ignore the fact that the wedges may overlap, so in what follows our estimate will be an upper bound of the area deleted. Secondly, we only focus on the evolution of one wedge, as the result can simply be multiplied through by 4 at the end. 
Thus initially we have one wedge and the area of the wedge is $A_{0}=\epsilon^{2} / \lambda_{0}$. After one iterate we have two wedges, the first is a copy (locally) of the original (corresponding to $\theta_{0}=(1 / 4$ or $3 / 4)-\omega$ ) and the other is the image of the first and has slope $\lambda_{1}$. So the total area deleted is $A_{1}=A_{0}+\epsilon^{2} / \lambda_{1}$. Iterating forward once more we have three wedges, the first two follow from the previous argument, whereas the third is the image of the second in the previous iteration. Thus $A_{2}=A_{1}+\epsilon^{2} / \lambda_{2}$. Generally we have $A_{n}=A_{n-1}+\epsilon^{2} / \lambda_{n}$ and so

$$
A_{n}=\epsilon^{2} \sum_{i=0}^{n} \lambda_{i}^{-1}=\frac{\epsilon^{2}}{\lambda_{0}}\left(1+\sum_{i=1}^{n} \frac{1}{p_{i}}\right) .
$$

Using the fact that $\sigma^{i}$ is a lower bound for $p_{i}$ we can thus write

$$
A_{n}<\frac{\epsilon^{2}}{\lambda_{0}}\left(1+\sum_{i=1}^{n}\left(\frac{1}{\sigma}\right)^{i}\right) .
$$

Since this is an upper bound we learn nothing about $A_{n}$ if the right hand side diverges. The sum on the right is a geometric series which converges if, and only if, $\sigma>1$. In this case we can write $A_{n} \simeq C \epsilon^{2}<2 \epsilon$ for $\epsilon$ sufficiently small. Hence for $\sigma>1$ we have non negligible area in the $\epsilon$ strip and we conclude that the box-counting dimension is 2 .

\subsection{Making the argument exact: piecewise linear SNAs}

In the example above, we assume that for $\epsilon$ sufficiently small the edges of the wedges are approximately linear. However, there are examples of SNAs where locally every piece of the SNA is linear - the so-called piecewise linear SNAs. We define a piecewise linear SNA to be an system exhibiting SNA where the non-linearity in the $x$ component is piecewise linear. As an example we take the slightly modified system

$$
\begin{aligned}
& x_{n+1}=2 f\left(x_{n}\right) g\left(\theta_{n}\right), \\
& \theta_{n+1}=\theta_{n}+\omega \quad(\bmod 1) .
\end{aligned}
$$

We take

$$
f(x)= \begin{cases}\alpha x, & |x| \leq 1 / \alpha \\ \operatorname{sign}(x), & |x|>1 / \alpha,\end{cases}
$$

and $g(\theta)=\cos (2 \pi \theta)$ as before. The system undergoes transition to SNA at $\alpha=1$, which can be verified using the same technique introduced in [8].

The upper bound for the area deleted from an $\epsilon$ strip $A_{n}$ for this system is the same as before - only more accurate, as the wedges are now practically linear in nature for $\epsilon$ sufficiently small. We conclude once more that the boxcounting dimension is 2 for $\alpha>1$. An interesting point to note is that for any initial value $x_{0}$ such that $\left|x_{i}\right|>1 / \alpha$ for some $i=0,1,2, \ldots$ we have that $f^{\prime}\left(x_{i}\right)=0$ and so the Lyapunov exponent $\lambda=-\infty$ giving a "superstable" attractor. This will always be the case for $\alpha>1$, because if we assume that $\left|x_{0}\right| \leq 1 / \alpha$ then under iteration of (2.25) we have $x_{i} \sim \alpha^{i} x_{0}$ from Lemma 1 , and hence eventually we will have $\left|x_{m}\right|>1 / \alpha$ for some $m$. We will call such an attractor a super strange non-chaotic attractor (SSNA).

We can however do even better than this by ensuring that all components of the system are piecewise linear. In [18] a similar piecewise linear system was studied of the form

$$
\begin{aligned}
x_{n+1} & =\alpha f\left(x_{n}\right) g\left(\theta_{n}\right), \\
\theta_{n+1} & =\theta_{n}+\omega \quad(\bmod 1) .,
\end{aligned}
$$

with $g(\theta)=\theta-1 / 2$ and $f(x)$ as above. Using the argument presented in [8] for systems of pinched skew product type we conclude that the attractor is strange. 


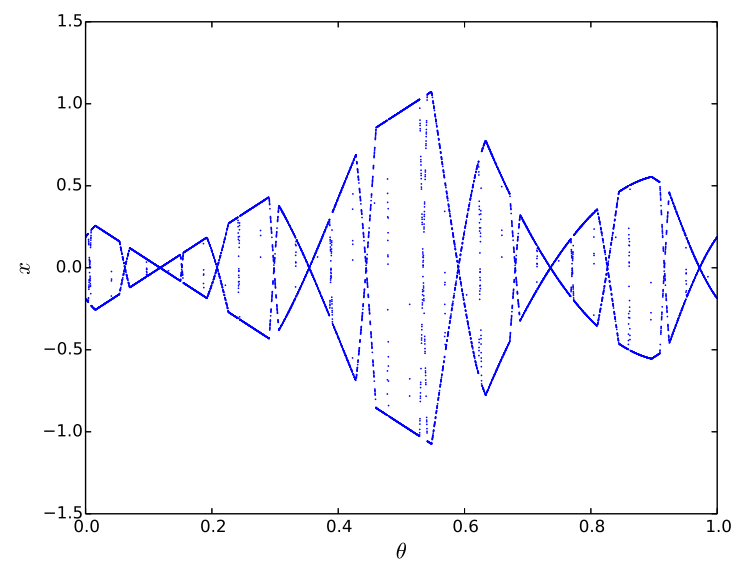

Figure 8: Strange non-chaotic attractor occurring for $\alpha=2.5$ in system (2.28)-(2.29).

A plot of the SNA obtained at $\alpha=2.5$ is shown in Figure 8. In [18] it is numerically observed that the transition to SNA occurs at $\alpha \simeq 2.33$. In fact, the precise value of this constant may be readily derived by calculation of the transverse Lyapunov exponent of $x=0$. Letting $F(x, \theta)=\alpha f(x) g(\theta)$ we have that this exponent is given by (using the ergodicity in $\theta$ )

$$
\begin{aligned}
\lambda_{t} & =\int_{0}^{1} \ln \left|\frac{\partial F}{\partial x}\right|_{x=0} \\
& =\int_{0}^{1} \ln \left|\alpha f^{\prime}(x) g(\theta)\right|_{x=0} \mathrm{~d} \theta \\
& =\int_{0}^{1} \ln \left|\alpha^{2}(\theta-1 / 2)\right| \mathrm{d} \theta \\
& =\frac{1}{\alpha^{2}} \int_{-\alpha^{2} / 2}^{\alpha^{2} / 2} \ln |y| \mathrm{d} y \\
& =\ln \left(\alpha^{2} / 2\right)-1 .
\end{aligned}
$$

The critical point at which $x=0$ loses stability is when $\lambda_{t}=0$, which corresponds to $\alpha_{c}=\sqrt{2 e}=2.331643 \ldots$ Using the methods described above we can show that the box-counting dimension becomes 2 for $\alpha>\alpha_{c}$.

In particular, using the same elementary straight linear geometry as before we can show that, in the limit $\epsilon \rightarrow 0$ we have that the slopes of the wedges satisfy

$$
\lambda_{n}=\lambda_{0} \prod_{i=1}^{n} \alpha^{2}|g((i+1) \omega)| .
$$

In Figure 9 we show the plot of the evolution of the initial area $\mathcal{A}_{0}=\{(\theta, x): 0 \leq \theta<1,-\infty<x<\infty\}$, for four iterates of system (2.28)-(2.29) and the wedges are clearly visible in the $\epsilon$ strip in similar fashion to Figure 2. Using the same argument as in Lemma 1 we can show that

$$
r_{n}=\left(\prod_{i=1}^{n}|g((i+1) \omega)|\right)^{1 / n} \rightarrow \frac{e^{-1}}{2}, \quad n \rightarrow \infty .
$$

We see numerically similar to before that $r_{n}>e^{-1} / 2$ and so an upper bound for the area deleted from the $\epsilon$ strip is

$$
A_{n}<\frac{\epsilon^{2}}{\lambda_{0}}\left(1+\sum_{i=1}^{n}\left(\frac{2}{\alpha^{2} e^{-1}}\right)^{i}\right) \text {. }
$$


The geometric series on the right will not converge unless $\alpha>\sqrt{2 e}$ and we conclude that $D_{0}=2$ for $\alpha>\alpha_{c}$. If $\alpha>\alpha_{c}$ then for any $\left|x_{0}\right| \leq 1 / \alpha$ we have $x_{i} \sim\left(\alpha^{2} e^{-1} / 2\right)^{i} x_{0}$ and so eventually $\left|x_{m}\right|>1 / \alpha$ for some $m$ which makes $\frac{\partial F}{\partial x}\left(\theta_{m}, x_{m}\right)=0$ and hence we have a SSNA.
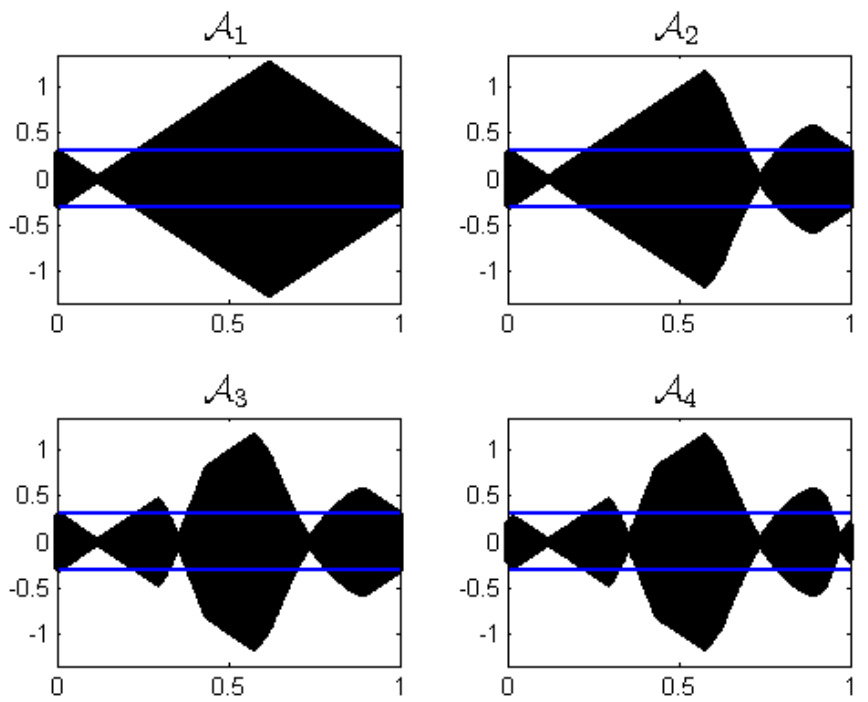

Figure 9: Successive iterates of the initial area $\mathcal{A}_{0}$ under (2.28)-(2.29).

\section{Generalising the result to a class of pinched skew-products}

This method may be applied to a whole class of "pinched skew-products", which we define following the work of Glendinning in [6] as a map of the form $P: X \rightarrow X$ with

$$
P(\theta, x)=(\theta+\omega, b(\theta, \mu) f(x, \mu)),
$$

where $\omega \in \mathbb{R} \backslash \mathbb{Q}, X=(\mathbb{R} \backslash \mathbb{Z}) \times \mathbb{R}$ and $\mu$ is a bifurcation parameter. We now make a number of assumptions on the function appearing in the second component of (3.1) as follows.

1. We assume that $b$ and $f$ are piecewise smooth functions and in addition that $f$ and $\mathrm{d} b / \mathrm{d} \theta$ are both bounded.

2. $f(0, \mu)=0$ and

$$
\lim _{\epsilon \rightarrow 0} \frac{f(\epsilon, \mu)}{\epsilon}
$$

exists for all $\mu$.

3. The function $b$ has at least one zero at $\tilde{\theta}$ (say), and that the number of zeros is finite.

The first of these assumptions ensures that the function in the second component of $P$ can be well approximated by a linear function in a neighbourhood of a point, which is required if our methodology is to work in calculating the fractal dimension.

The line $\mathcal{L}=\{(\theta, x): x=0\}$ is invariant. If the transverse Lyapunov exponent of $\mathcal{L}$ is positive then $\mathcal{L}$ is unstable and the pinching effect occurs, creating the approximately linear wedges within an $\epsilon$ strip required for our analysis to be valid. 
Let the $m$ roots of $b(\theta, \mu)$ be given by $\tilde{\theta}_{i}, i=1, \ldots, m$. Then there will be tips of wedges located at $\tilde{\theta}_{i}+\omega$ after the first iterate of the initial area $\mathcal{A}_{0}=\{(\theta, x): 0 \leq \theta<1,-\infty<x<\infty\}$. Each of these wedges will have a slope $\lambda_{0, i}$ which can be calculated (although it is not necessary to the analysis) using the formula

$$
\lambda_{0, i}=\max _{-\infty<x<\infty}\left\{\left|\frac{\partial F}{\partial \theta}\left(\tilde{\theta}_{i}, x, \mu\right)\right|\right\},
$$

where $F(\theta, x, \mu)=b(\theta, \mu) f(x, \mu)$. If $b(\theta, \mu)$ is periodic on the unit interval, then many of these initial slopes will trivially be duplicated which simplifies matters, as is the case in $(2.1)-(2.2)$ where $b(\theta, \mu)=\cos (2 \pi \theta)$ is symmetric about $1 / 2$. We can now write

$$
\lambda_{n, i}=\lambda_{0, i}\left|l_{\mu}^{n}\right| \prod_{i=1}^{n}\left|b\left(\theta_{0, i}+i \omega\right)\right|
$$

where

$$
l_{\mu}=\lim _{\epsilon \rightarrow 0} \frac{f(\epsilon, \mu)}{\epsilon},
$$

a limit that we have assumed exists. Using the ergodicity in $\theta$ we can say that $s_{n}=\left(\prod_{i=1}^{n}|b(\theta+i \omega)|\right)^{1 / n} \rightarrow e^{\chi}$ where

$$
\chi=\int_{0}^{1} \ln |b(\theta, \mu)| \mathrm{d} \theta
$$

This means that $\lambda_{n, i} \sim\left|l_{\mu} e^{\chi}\right|^{n}$ and so an upper bound on the area deleted in the evolution of a single wedge can be given by

$$
A_{n}=C \epsilon^{2}\left(1+\sum_{i=1}^{n}\left(\frac{1}{\left|l_{\mu} e^{\chi}\right|}\right)^{i}\right)
$$

for some constant $C$. This will converge if, and only if, $\left|l_{\mu} e^{\chi}\right|>1$. If this is the case then the box-counting dimension is 2 , and a condition on $\mu$ can typically be derived to ensure this is the case. For example, for system (2.1)-(2.2) we let $b(\theta, \mu)=\cos (2 \pi \theta)$ and $f(x, \mu)=2 \mu \tanh (x)$. This gives us $l_{\mu}=2 \mu$ and $e^{\chi}=0.5$, and so $\left|l_{\mu} e^{\chi}\right|>1$ implies $\mu>1$. In system (1.1)-(1.2) we can easily verify that the dimension is 2 for $\kappa>2$ using this method.

For system (2.28)-(2.29) we can let $b(\theta, \mu)=\theta-1 / 2$ and $\tilde{f}(x, \mu)=\mu f(x, \mu)$ with $f$ as defined in (2.27). Then $e^{\chi}=e^{-1} / 2$ and $l_{\mu}=\mu^{2}$ and hence the box-counting dimension is 2 for $\mu>\sqrt{2 e}$ which is the value derived in Subsection 2.1 as the critical point of transition to SNA for this system.

Finally, we take

$$
\begin{aligned}
b(\theta, \mu) & =|\cos (2 \pi \theta)| \\
f(x, \mu) & =2 \mu x(1-x),
\end{aligned}
$$

so that we have a quasiperiodically forced logistic map. The above method then tells us that the dimension of the attractor is 2 for $\mu>1$. A plot of the attractor for $\mu=2$ is given in Figure 10. Numerical calculation of the Lyapunov exponent indicates that the attractor is non-chaotic (as shown in Figure 11), and once again we can apply the arguments in [8] to confirm its strangeness. 


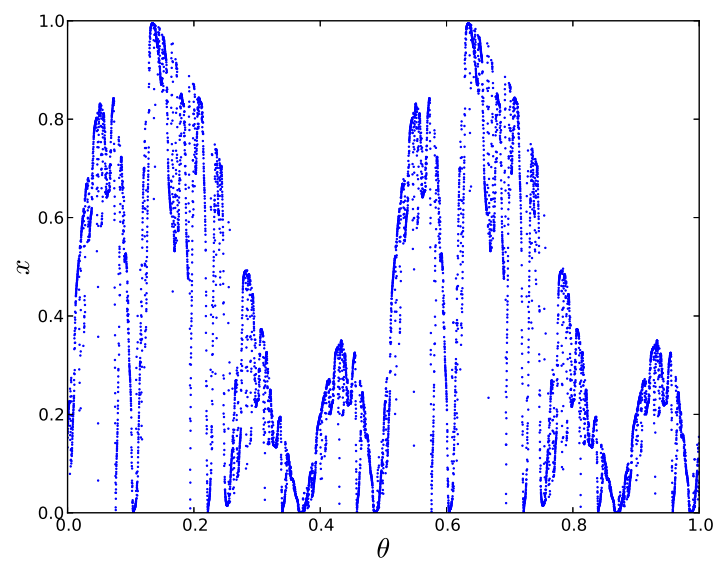

Figure 10: Plot of the attractor for the quasiperiodically forced logistic map (3.8)-(3.9) when $\mu=2$.

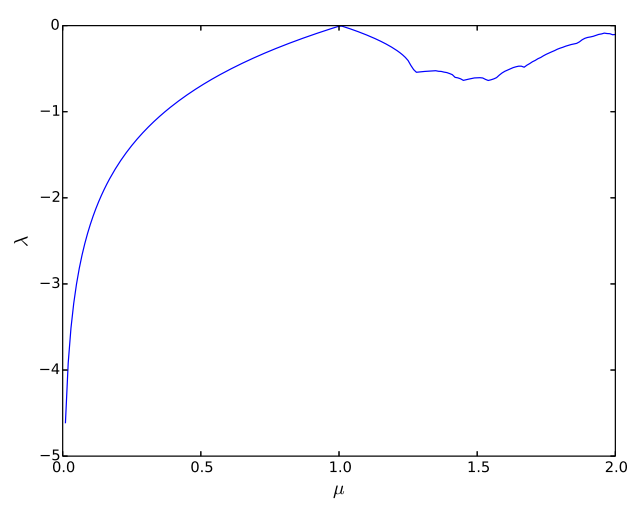

Figure 11: Plot of a numerical evaluation of the Lyapunov exponent $\lambda$ against the bifurcation parameter $\mu$.

\section{Dimensions of critical SNAs}

Section's 2 and 3 also enlighten us as to the behaviour of the systems at the critical point of transition to SNA. For example let us consider the system (2.1)-(2.2) at the critical point of transition to SNA at $\sigma_{c}=1$. A plot of the attractor at the critical point is shown in Figure 12. 


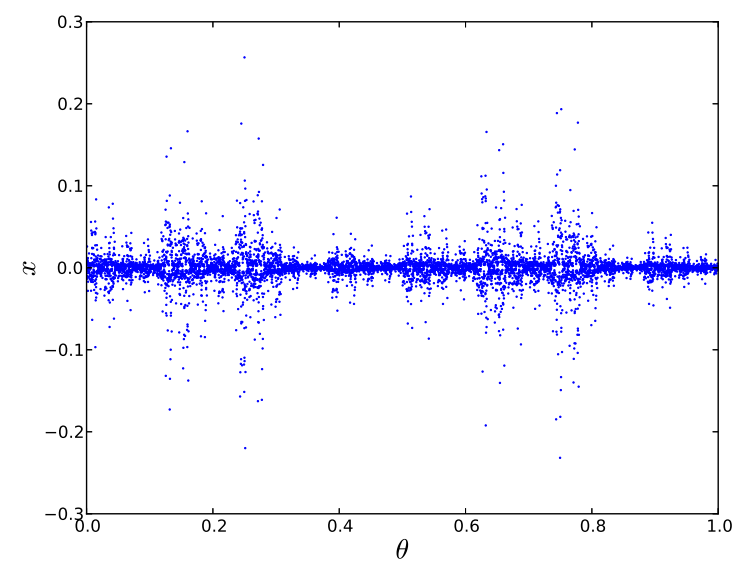

Figure 12: Strange non-chaotic attractor occurring at $\sigma_{c}=1.0$ in system (2.1)-(2.2).

Now, according to (2.22), at the critical point the slopes of successive iterates of the wedges do not grow exponentially. Of course, using the fact that $\tilde{\epsilon}(n) \propto n^{-1}$ and the results from Section 2 we could conclude that $\lambda_{n}$ is proportional to a constant asymptotically, but from Figure 6 we see that the actual decay fluctuates either side of the theoretical decay, and these fluctuations are magnified upon multiplication by $2^{n}$ giving linearly growing oscillations on a logarithmic scale. A plot of $\lambda_{n}$ against $n$ is presented in Figure 13 confirming this. Hence there are infinitely many wedges whose area is non-negligible, and thus, due to the fact that the tips of the wedges explore the $\theta$ axis ergodically, the area in the $\epsilon$ strip should decay to zero asymptotically, indicating that the box counting dimension may not be 2 .

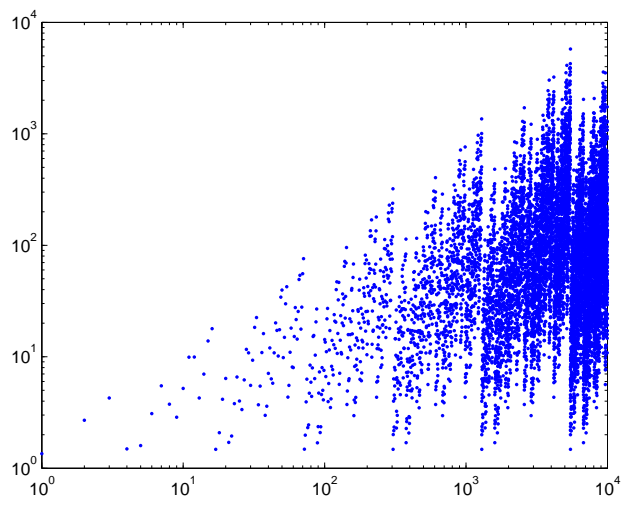

Figure 13: Plot of $\lambda_{n}$ vs. $n$ for $\sigma_{c}=1$ showing the existence of finite slope sizes for arbitrarily large $n$.

However, the dimension is not necessarily equal to 1 either. In [14] a renormalization analysis of the dynamics at the critical point reveals the self-similarity of the attractor at repeatedly smaller scales, and thus its fractal nature. Hence it is reasonable to expect that the dimension lies between 1 and 2 .

We have numerically calculated the box-counting dimension using boxes of size $h=1 / 2^{3}, \ldots, 1 / 2^{8}$ with $2 \times 10^{6}$ iterations for the GOPY model. The resulting plot and line of best fit is shown in Figure 14. The resulting plot is convincingly linear and has slope $\simeq 1.5$ (to $1 \mathrm{~d}$.p) which is our estimate of the fractal dimension. We also find the same approximate value of $D_{0}$ for the critical SNA of the piecewise linear system (2.25)-(2.26). 
For the piecewise linear system (2.28)-(2.29) from Section 4 we have calculated that the box-counting dimension at $\alpha_{c}=\sqrt{2 e}$ is $D_{0} \simeq 1.4$. Finally we note that a numerical calculation of the box-counting dimension for quasiperiodically forced logistic map (3.8)-(3.9) at the critical point $\mu=1$ gives $D_{0}=1$.

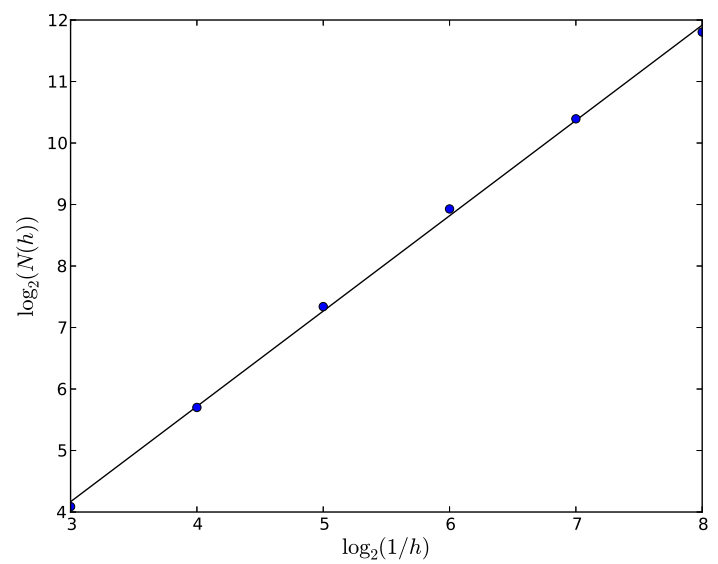

Figure 14: Plot of $\log _{2}(1 / h)$ vs. $\log _{2}(N(h))$ and the line of best fit.

\section{Conclusion}

We have modified the approach first used in [4] to provide strong evidence that the box counting dimension of a structurally stable "pinched" strange non-chaotic attractor (SNA) is equal to 2 . The result is obtained by examining the evolution of the area spanned by the $x$ and $\theta$ directions under iterations of the system within a small $\epsilon$ strip around the $\theta$ axis. Using a combination of techniques including a renormalization analysis, we are able to show that an upper bound of the area deleted is given by some constant multiple of $\epsilon^{2}$, indicating that for $\epsilon$ small enough the area remaining is non-negligible.

This argument can be made more accurate for piecewise linear SNAs. We used our new approach to verify that the piecewise linear SNA studied in [18] has dimension 2 past the critical bifurcation point, which we derived through both consideration of the transverse Lyapunov exponent of $x=0$ and our method of dimension calculation. Indeed, one of the benefits of this method is that the calculation of the dimension is dependent on the bifurcation parameter and so can be used to calculate the point of transition from a 1D attractor to a $2 \mathrm{D}$ attractor i.e. the point of transition to SNA. The piecewise linear SNAs under consideration are also of interest as they give rise to attractors which are super-stable (their Lyapunov exponent is $-\infty$ ) and we have dubbed these super strange non-chaotic attractors (SSNAs)!

Finally, we have provided evidence that the box-counting dimension of an SNA at a critical point is not typically equal to 2, but rather lies between 1 and 2. Numerical calculation of the dimension for several examples of critical SNAs adds further weight to this argument.

\section{References}

[1] L N C Adamson and A H Osbaldestin, The non-smooth pitchfork bifurcation: A renormalization analysis, Submitted to Dynamical Systems Journal.

[2] _ Renormalisation of correlations in a barrier billiard: Quadratic irrational trajectories, Physica D: Nonlinear Phenomena 270 (2014), 30-45. 
[3] J R Chapman and A H Osbaldestin, Self-similar correlations in a barrier billiard, Physica D 180 (2003), no. 1, $71-91$.

[4] M Ding, C Grebogi, and E Ott, Dimensions of strange nonchaotic attractors, Physics Letters A 137 (1989), no. $4,167-172$.

[5] U Feudel, A S Pikovsky, and M A Zaks, Correlation properties of a quasiperiodically forced two-level system, Physical Review E 51 (1995), no. 3, 1762-1769.

[6] P Glendinning, Global attractors of pinched skew products, Dynamical Systems: An International Journal 17 (2002), no. 3, 287-294.

[7] _ The nonsmooth pitchfork bifurcation, Discrete \& Continuous Dynamical Systems Series B 6 (2004), no. $4,457-464$.

[8] C. Grebogi, E. Ott, S. Pelikan, and J. A Yorke, Strange attractors that are not chaotic, Physica D 13 (1984), no. $1,261-268$.

[9] M Gröger and T Jäger, Dimensions of attractors in pinched skew products, Communications in Mathematical Physics 320 (2013), no. 1, 101-119.

[10] G Keller, A note on strange nonchaotic attractors, Fundamenta Mathematicae 151 (1996), no. 2, 139-148.

[11] J A Ketoja and I I Satija, Self-similarity and localization, Physical Review Letters 75 (1995), no. 14, 2762-2765.

[12] S P Kuznetsov, Generalized dimensions of the golden-mean quasiperiodic orbit from renormalization-group functional equation, Regular and Chaotic Dynamics 10 (2005), no. 1, 33-38.

[13] S P Kuznetsov and A H Osbaldestin, Generalized dimensions of feigenbaum's attractor from renormalizationgroup functional equations, Regular and Chaotic Dynamics 7 (2002), no. 3, 325-330.

[14] S P Kuznetsov, A S Pikovsky, and U Feudel, Birth of a strange nonchaotic attractor: A renormalization group analysis, Physical Review E 51 (1995), no. 3, R1629-R1632.

[15] A G Leonov and N V Kuznetsov, Time-varying linearization and the Perron effects, International Journal of Bifurcation and Chaos 17 (2007), no. 04, 1079-1107.

[16] B D Mestel and A H Osbaldestin, Renormalization analysis of correlation properties in a quasiperiodically forced two-level system, Journal of Mathematical Physics 43 (2002), 3458-3483.

[17] __ Golden mean renormalization for a generalized Harper equation: The Ketoja-Satija orchid, Journal of Mathematical Physics 45 (2004), 5042-5075.

[18] S S Negi and R Ramaswamy, A plethora of strange nonchaotic attractors, Pramana 56 (2001), no. 1, 47-56.

[19] A H Osbaldestin and L N C Adamson, Chaotic correlations in barrier billiards with arbitrary barriers, Journal of Physics A: Mathematical and Theoretical 46 (2013), no. 24, 245101.

[20] A S Pikovsky and U Feudel, Characterizing strange nonchaotic attractors, Chaos: An Interdisciplinary Journal of Nonlinear Science 5 (1995), no. 1, 253-260. 